\title{
Relationship Between Catalyst Composition and Carbon Morphology
}

\author{
X. Z. Liao,* A. Serquis, ${ }^{*}$ Q. X. Jia, ${ }^{*}$ D. E. Peterson, ${ }^{*}$ Y. T. Zhu,* and H. F. Xu** \\ * Los Alamos National Laboratory, Division of Materials Science and Technology, Los Alamos, \\ NM 87545 \\ ** University of New Mexico, Department of Earth \& Planetary Sciences, Albuquerque, NM 87131
}

Catalytic chemical vapor deposition with bi-metallic catalysts has been used to produce carbon nanotubes (CNTs). The catalyst composition affects significantly the CNT growth, but the effect has not been quantitatively reported. In this study, we used high-resolution transmission electron microscopy, X-ray energy-dispersive spectroscopy (EDS), and elemental mapping using electron energy filtering imaging to correlate quantitatively the compositions of individual Co-Mo catalyst particles with the morphologies of corresponding CNTs.

A sol-gel method was used for Co-Mo catalyst preparation. CNTs were grown at $700^{\circ} \mathrm{C}$ using $\mathrm{CO}$ source. TEM investigations were carried out using a JEOL $2010 \mathrm{~F}$ operated at $200 \mathrm{kV}$. Quantitative EDS measurement was carried out using $\mathrm{Co}-\mathrm{K} \alpha$ line and Mo-L $\alpha$ line.

Extensive investigations revealed three kinds of multi-walled carbon morphologies: (1) long CNTs, with lengths from about 0.1 micron to a few microns, tipped with catalyst particles partially exposed to environment (see Fig. 1(a)); (2) short CNTs with lengths normally less than $50 \mathrm{~nm}$ and with catalyst particles fully encapsulated by graphene sheets (see Fig. 2(a)); and (3) spherical, onionshaped graphite with a catalyst particle at the center (see Fig. 3(a)). The fact that most long CNTs observed have their catalyst particles partially exposed indicates that the direct contact of catalyst surface with carbon source is essential for continuous CNTs growth. When a catalyst particle is fully encapsulated by layers of graphene sheets, the carbon supply route is cut, and the CNT growth stops, resulting in a short CNT, as shown in Fig. 2(a).

EDS results indicate that the compositions of individual catalyst particles can generally be categorized into three groups. In general, there is a very clear relationship between the catalyst composition and carbon morphology. Catalyst particles with 5-15 at.\% Co tend to produce long CNTs, those with 40-45 at.\% Co tend to produce short CNTs, and those with 85-98 at.\% Co tend to produce onionated morphology.

The elemental mapping results in Figs. 1 to 3, on the other hand, suggest that the composition in a catalyst is not uniformly distributed. Severe heterogeneity in Co (or Mo) distribution in an individual particle was found to affect the carbon morphology. For example, a few particles with average Co composition of around 50 at.\% produced long CNTs instead of short ones. However, elemental mapping images of these particles suggest that the Co content near the exposed particle surface is much lower than the average Co content, implying that it is the Co content on the exposed particle surface, instead of the average Co content in the whole particle, that determines the carbon morphology.

The above observations will be discussed in terms of CNT growth mechanism and the catalytic activity of $\mathrm{Co}$ and Mo. The combined effect of catalyst composition and other growth conditions (e.g. CO partial pressure) on CNT growth will also be discussed. 


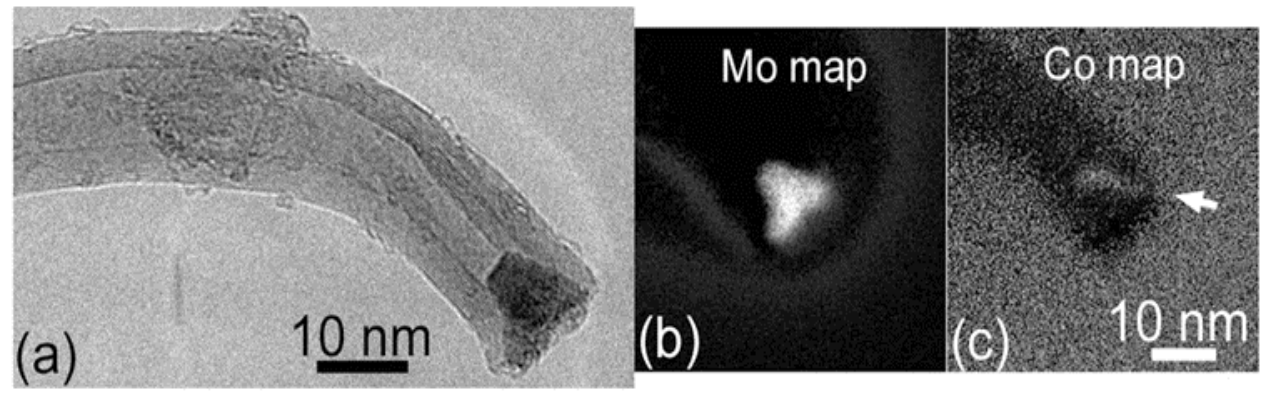

Figure 1 (a) An image of a typical long multi-walled CNT; (b) Mo elemental map; and (c) Co elemental map showing more Co at one edge of the particle as indicated by an arrow.

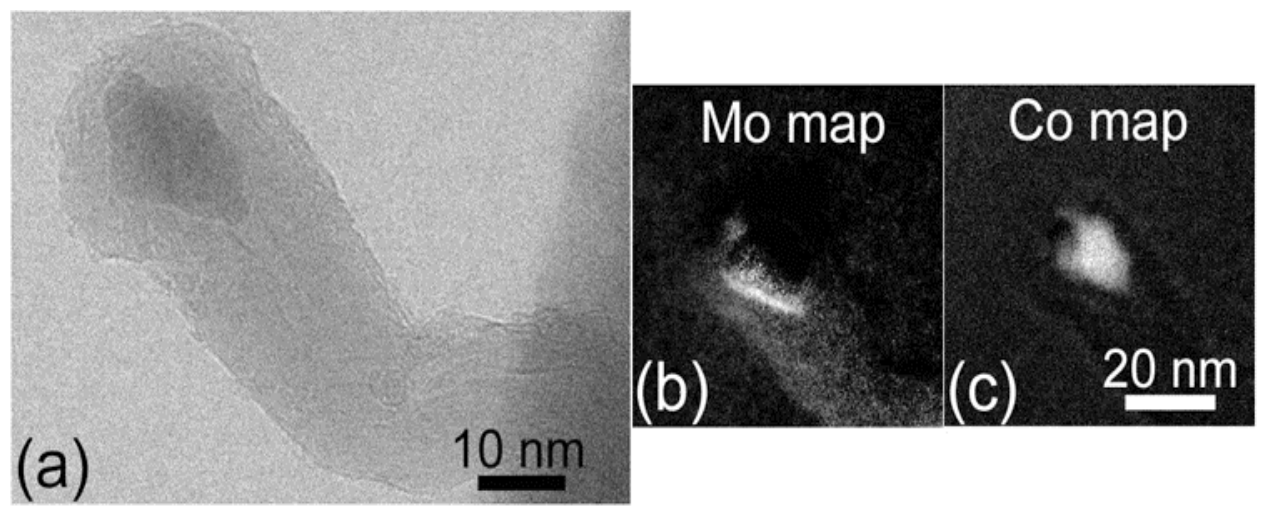

Figure 2 (a) An image of a typical short multi-walled CNT with a catalytic particle fully encapsulated by the graphene layers; (b) Mo elemental map; and (c) Co elemental map.

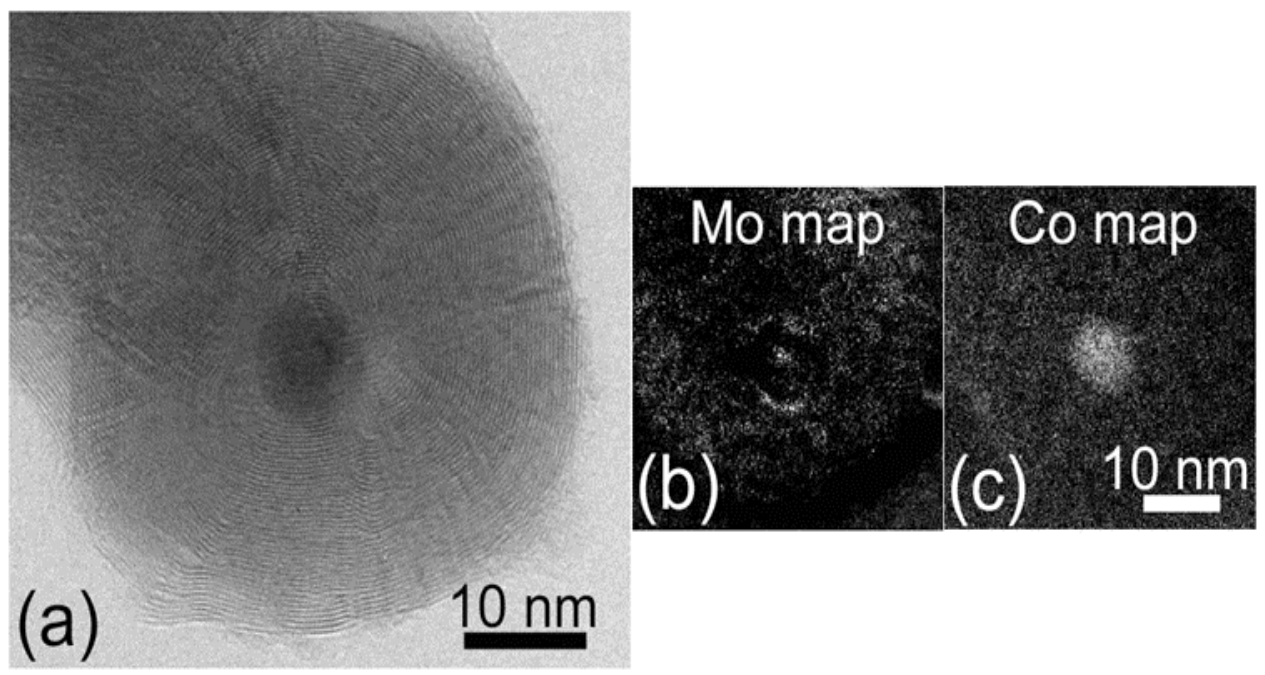

Figure 3 (a) A high-resolution TEM showing a typical onionated multi-walled CNT with a catalytic particle located at the center of the "onion"; (b) Mo elemental map; and (c) Co elemental map. 